\title{
Chemotherapy vs tamoxifen in platinum-resistant ovarian cancer: a phase III, randomised, multicentre trial (Ovaresist)
}

Kristina Lindemann ${ }^{* 1,2,3}$, Emma Gibbs ${ }^{4}$, Elisabeth Åvall-Lundqvist ${ }^{5,6}$, Rene dePont Christensen ${ }^{7}$, Kathrine Woie ${ }^{8}$, Marten Kalling ${ }^{9}$, Annika Auranen ${ }^{10}$, Seija Grenman ${ }^{11,12}$, Thomas Hoegberg ${ }^{13}$, Per Rosenberg ${ }^{14}$, Tone Skeie-Jensen ${ }^{1}$, Elisabet Hjerpe ${ }^{7}$, Anne Dørum ${ }^{1}$, Val Gebski ${ }^{4}$ and Gunnar Kristensen ${ }^{1,15}$ ${ }^{1}$ Department of Gynecologic Oncology, Norwegian Radium Hospital, Oslo University Hospital, Oslo, Norway; ${ }^{2}$ NHMRC Clinical Trials Centre, University of Sydney, Camperdown, NSW, Australia; ${ }^{3}$ Department of Medical Oncology, Westmead Hospital, Crown Princess Mary Cancer Centre, Wentworthville, NSW, Australia; ${ }^{4}$ NHMRC Clinical Trials Centre, University of Sydney, Camperdown, NSW 2050, Australia; ${ }^{5}$ Department of Oncology and Department of Clinical and Experimental Medicine, Linköping University, Linköping, Sweden; ${ }^{6}$ Department of Oncology and Pathology, Karolinska Institutet, Stockholm, Sweden; ${ }^{7}$ Research Unit of General Practice, Institute of Public Health, University of Southern Denmark, Odense, Denmark; ${ }^{8}$ Department of Gynecologic Oncology, Haukeland University Hospital, Bergen, Norway; ${ }^{2}$ Department of Gynecologic Oncology, Skane University Hospital, Lund, Sweden; ${ }^{10}$ Department of Obstetrics and Gynecology, Tampere University Hospital, Tampere, Finland; ${ }^{11}$ Department of Obstetrics and Gynecology, Turku University Hospital, Turku, Finland; ${ }^{12}$ University of Turku, Turku, Finland; ${ }^{13}$ Department of Cancer Epidemiology, Skane University Hospital Lund, Lund, Sweden; ${ }^{14}$ Department of Oncology, Linköping University Hospital, Linköping, Sweden and ${ }^{15}$ Institute for Cancer Genetics and Informatics, Oslo University Hospital, Oslo and University of Oslo, Oslo, Norway

Background: Chemotherapy in platinum-resistant ovarian cancer (PROC) aims for palliation and prolonging of progression-free survival (PFS). This study compares Health-related Quality of Life (HROoL) and efficacy between single-agent chemotherapy and tamoxifen in PROC.

Methods: Patients with PROC were randomised (2:1) to chemotherapy (weekly paclitaxel $80 \mathrm{mg} \mathrm{m}^{-2}$ or four weekly pegylated liposomal doxorubicin $40 \mathrm{mg} \mathrm{m}^{-2}$ ) or tamoxifen $40 \mathrm{mg}$ daily. The primary end point was HRQoL. Secondary end points were PFS by RECIST and overall survival (OS).

Results: Between March 2002 and December 2007, 156 and 82 patients were randomised to chemotherapy and tamoxifen, respectively. In the chemotherapy arm, a significantly larger proportion of patients experienced a worsening in their social functioning. There was no difference in the proportion of patients experiencing improvement of gastrointestinal symptoms. Median PFS on tamoxifen was 8.3 weeks (95\% Cl, 8.0-10.4) compared with 12.7 weeks (95\% Cl, 9.0-16.3) on chemotherapy (HR, $1.54 ; 95 \% \mathrm{Cl}, 1.16-2.05$; log-rank $P=0.003)$. There was no difference in OS between the treatment arms.

Conclusions: Patients on chemotherapy had longer PFS but experienced more toxicity and poorer HRQoL compared with tamoxifen. Control over gastrointestinal symptoms was not better on chemotherapy. These data are important for patient counselling and highlight the need to incorporate HRQoL end points in studies of PROC.

*Correspondence: Dr K Lindemann; E-mail: klinde@ous-hf.no

Received 1 July 2016; revised 19 November 2016; accepted 10 December 2016; published online 24 January 2017

(c) 2017 Cancer Research UK. All rights reserved 0007-0920/17 
Chemotherapy for patients with recurrent ovarian cancer remains palliative, with the aim to improve quality of life and prolong time to progression. The platinum-free interval is of predictive significance as responses to re-exposure to platinum are less likely with shorter time since last platinum-based chemotherapy (Gore et al, 1990; Markman et al, 1991). Ultimately, most patients with recurrent disease will develop platinum resistance. In this setting, combination therapies are associated with increased toxicity without advantage in overall survival (OS) (Buda et al, 2004; Sehouli et al, 2008; Lortholary et al, 2012), and single-agent therapies have been favoured. The most active agents are pegylated liposomal doxorubicin (PLD), weekly paclitaxel, gemcitabine and topotecan (ten Bokkel Huinink et al, 1997; Gordon et al, 2001; Mutch et al, 2007; Vergote et al, 2010) with response rates between 8.3 and $21 \%$. Only recently the combination of bevacizumab and single-agent chemotherapy has been reported to significantly increase progression-free survival (PFS) compared with chemotherapy alone (Pujade-Lauraine et al, 2014). Still, treatment with chemotherapy is associated with side effects like neutropenia, alopaecia, neuropathy and palmar-plantar erythrodysesthesia (PPE) and these have been shown to impair patient's quality of life (Lakusta et al, 2001; Stiggelbout and de Haes, 2001; Le et al, 2004). Hormonal treatment has been considered a worthwhile alternative to chemotherapy due to its limited side effects and simple dosing. The antitumour activity of tamoxifen is believed to be mediated by competitive binding to oestrogen receptors, but a direct antiproliferative effect of oestrogen antagonists and progesterone has also been suggested (Batra and Iosif, 1996). To our knowledge, there is to date only one comparative study of endocrine treatment (leuprorelin) vs chemotherapy (treosulfan) (du Bois et al, 2002). This study was stopped prematurely due to concerns about the lack of efficacy. For tamoxifen, phase II studies have reported response rates around 10-13\% (Markman et al, 1996; Williams et al, 2010) with stabilisation of disease in $32 \%$ of the patients, which is not dissimilar to the results of chemotherapy in this population (Hatch et al, 1991).

In the context of platinum-resistant ovarian cancer (PROC), the trade-offs between clinical benefit and quality of life are important and the performance of tamoxifen against chemotherapy in this patient group has not been previously investigated in a phase III setting.

This is a phase III randomised study comparing single-agent chemotherapy with tamoxifen in patients with PROC. Preliminary results were presented at the 2008 Annual Meeting of the American Society of Clinical Oncology.

\section{PATIENTS AND METHODS}

The study was designed and carried out in accordance with good clinical practice, the declaration of Helsinki and national laws. Local ethics committee of each participating centre approved the study. All patients provided written informed consent before study entry. The study is registered at ClinicalTrials.gov with Identifier: NCT02728622.

Eligibility criteria, randomisation and quality assurance. Patients with histologically confirmed invasive epithelial ovarian, fallopian tube or peritoneal cancer, being resistant to treatment with platinum and a taxane (in a three-weekly regimen), either given in combination or sequentially, were eligible. Resistance was defined as one of the following. (1) Clinical progression during or within 6 months after end of treatment for primary disease or relapse. (2) Stable disease after six courses of chemotherapy for primary disease or relapse. (3) Doubling of CA 125 to at least $70 \mathrm{IU} \mathrm{ml}{ }^{-1}$ within 3 months after end of treatment for primary disease or relapse.
Patients were to be aged $\geqslant 18$ years, have ECOG performance status $\leqslant 2$, and have adequate liver, renal and bone marrow function. Exclusion criteria were symptomatic brain metastases, active infection or other severe underlying medical condition.

Randomisation was performed by computerised minimisation techniques. Patients were randomised $1: 2$ to either hormonal treatment or chemotherapy of investigator's choice.

The responsible study office (NSGO-CTU) followed the protocol-defined guidelines on central monitoring and reviewed case report forms for completeness and consistency.

Treatment plan and dose modifications. Patients randomised to hormonal treatment received oral tamoxifen $40 \mathrm{mg}$ daily. Patients randomised to chemotherapy received either paclitaxel $80 \mathrm{mg} \mathrm{m}^{-2}$ as a 1-h infusion, given every 7 days or PLD $40 \mathrm{mg} \mathrm{m}^{-2}$ given every 4 weeks. The first dose was infused over $2 \mathrm{~h}$; later doses were infused over $1 \mathrm{~h}$. Chemotherapy was administered with appropriate premedication.

Study specific guidelines were followed for dose delay and dose reduction for haematologic and non-haematologic toxicity and for supportive care use.

The use of supportive G-CSF treatment was not recommended instead of dose reduction but was left to the investigator's discretion.

Assessment of PROs, efficacy and follow-up. Health-related Quality of Life (HRQoL) was evaluated using the European Organization for Research and Treatment of Cancer (EORTC) quality-of-life questionnaire (QLQ-C30, version 3.0) and the specific module for ovarian cancer (QLQ-OV-28, version 1.0). Health-related Quality of Life was assessed at baseline and every 8 weeks ( \pm 2 weeks) during treatment and every 12 weeks $( \pm 2$ weeks) thereafter until patient death or withdrawal. In this analysis, questionnaires from all assessments until disease progression were analysed.

Tumour measurements according to Response Evaluation Criteria in Solid Tumors (RECIST) (Therasse et al, 2000) were recorded at baseline and every 8 weeks using the same tumour assessment technique throughout the study. Treatment was discontinued in case of clinical tumour progression or deterioration of performance status not being compatible with continued treatment. In case of complete clinical remission, treatment could be terminated after two more courses of PLD or six more courses of paclitaxel.

In case of progression on protocol treatment, a crossover from tamoxifen to chemotherapy and from chemotherapy to tamoxifen was recommended if continued treatment was in the best interest of the patient.

Adverse events and toxicity were graded during each cycle by the study investigator according to the National Institute Common Toxicity Scale version 1.0 (Cancer Therapy Evaluation Program, 1992). Toxicities were evaluated using the worst score per toxicity in each patient.

Date of last follow-up was the 5 January 2009.

Statistical analyses. The primary end point was evaluation of HRQoL in both treatment arms. Secondary end points were PFS and OS.

The study was designed in 2002 when detailed aspects of HRQoL outcomes and sample size were described in general rather than in specific terms in the protocol. As the analysis of the primary end point HRQoL was not adequately prespecified in the protocol, the results presented here are considered secondary analyses. Nevertheless, the study was conducted to investigate whether the less toxic but potentially less effective treatment with tamoxifen would demonstrate an improvement in HRQoL compared with the presumably more toxic treatment with chemotherapy. In exploratory analyses of HRQoL, we assessed 
quality-adjusted survival (QAS) as a measure to address this research question.

In the original protocol, the sample size for the study was based on detecting a difference in HRQoL and also in PFS, with no adjustment for type I error. The power calculation for HRQoL was based on detecting an improvement of 1 point on the 7-point Likert scale; however, the version of QLQ-C30 used in the study did not present items on a 7-point Likert scale. For PFS, it was powered to detect at least a $54 \%$ increase in the risk of progression (hazard ratio (HR) of 1.54) on tamoxifen assuming a median time to progression of 13 weeks for those receiving tamoxifen and 20 weeks for those receiving chemotherapy. Based on a $2: 1$ randomisation, a 3-year accrual and 1-year follow-up, a sample size of 223 patients was required to detect this difference. Power calculation should ideally have been based on the primary end point, change in HRQoL, alone.

PFS was defined as the time from randomisation to disease progression or death from any cause. Overall survival was defined as the time from randomisation to death from any cause. Response was recorded as best response on treatment.

Efficacy and HRQoL analyses were based on the intent-to-treat population of eligible patients. Analysis of toxicities was based on the safety population (all patients who received at least one dose of study treatment). The scoring manuals from EORTC were applied to the HRQoL data to calculate scales for each instrument (Fayers et al, 2001). The abdominal/gastrointestinal (GI) symptom subscale comprised items 1-6 of the EORTC QLQ-OV28. The chemotherapy side effects subscale comprised items 13-17 and hormonal side effects items 18 and 19. Compliance of HRQoL was calculated using all patients, who had not progressed or died prior to the time point as the denominator.
All analyses of HRQoL questionnaires were performed using a mixed model for repeated measures with time by treatment interaction, together with the appropriate baseline scores, treatment allocation and time point. HRQoL subscales were presented for patients up to 30 days after their end of treatment day. HRQoLs were reassigned a visit based on the date of completion. On treatment this was any QoL within an 8 week window.

For the QAS analysis, the weights of McKenzie (McKenzie and van der Pol, 2009) were applied to the QLQ-C30 to estimate EQ5D utility. Survival probabilities were calculated using the area under the curve with an appropriate truncation time point of 43 weeks. An estimation of the QAS was calculated with a 95\% CI and $P$-value using bootstrapping methods with a 1000 replications. As visits were scheduled every $12 \pm 2$ weeks, QAS included all patients with at least one HRQoL measurement $12+2$ weeks prior to the date of progression or $12+2$ weeks after if no HRQoL prior to PD was available.

In responder analysis we studied the proportion of patients with a clinically significant change in HRQoL score on a particular scale compared with their baseline score. An improvement was defined as at least $15 \%$ increase with a $10 \%$ increase used in sensitivity analysis (Osoba et al, 2005; Stockler et al, 2014). Patients with a HRQoL decrease of $15 \%$ or more ( $10 \%$ in sensitivity analysis) were classified as 'worsened', whereas a change within those ranges was classified as 'no change'. Chi-square test was used to determine the statistical significance of differences between the treatment arms. Subgroup analysis was performed for the GI symptom scale in relationship to patients' severity of GI symptoms at baseline. Category boundaries were determined using median score at baseline for the dividing patients into those with high and low baseline symptoms.

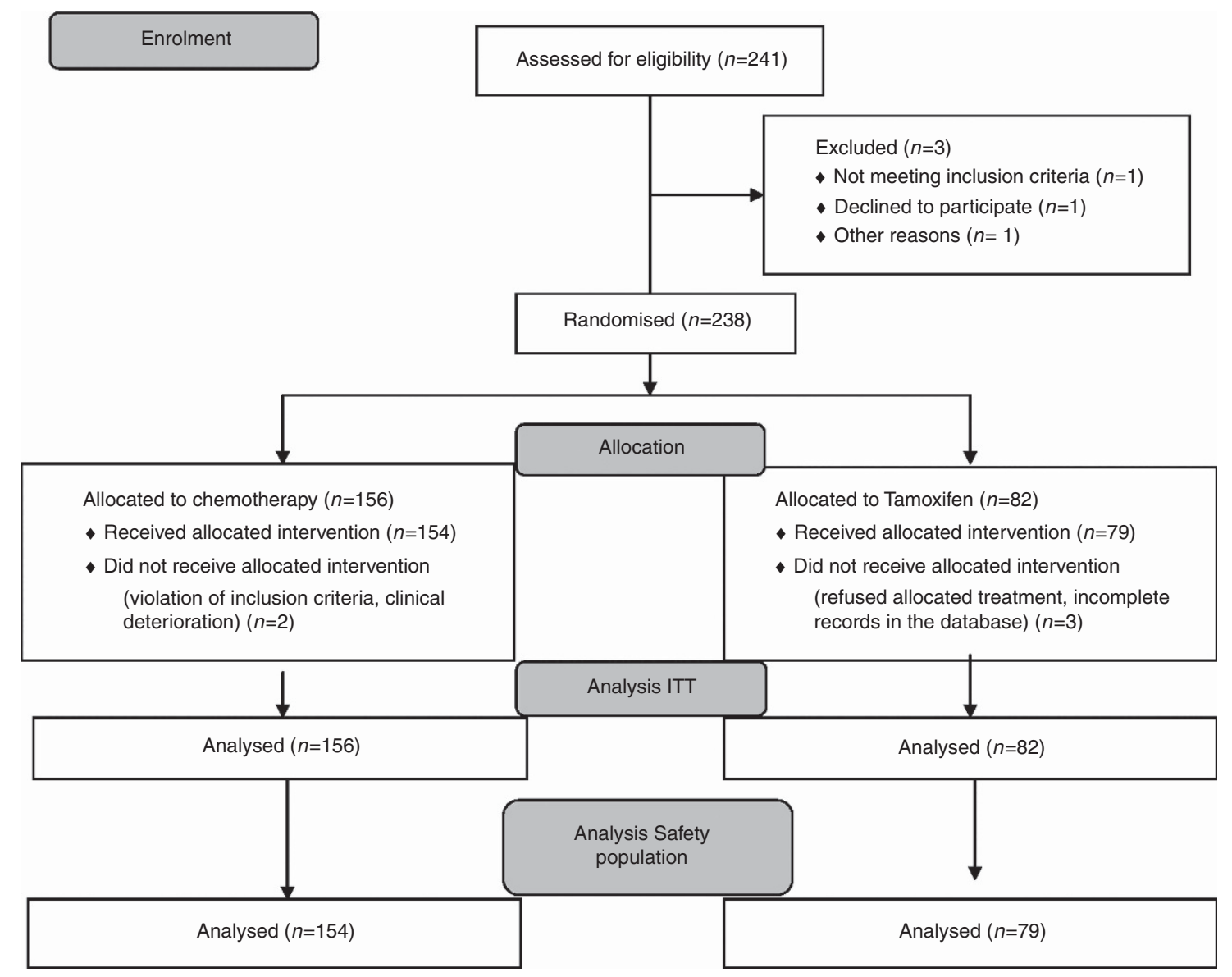

Figure 1. CONSORT diagram: enrolment, randomization and treatment. 
Time-to-event data were analysed using the Kaplan-Meier method, and the log-rank test was used to compare the distributions between groups. Hazard ratios with 95\% CIs were estimated using the Cox proportional hazards model. Sensitivity analysis used a stratified log-rank test stratifying for the a priori defined factor of progression during or after discontinuation of chemotherapy.

Toxicity was described for each group separately and proportions compared by the Wilcoxon-Mann-Whitney test.

SAS statistical package, version 9.3, was used for the analysis. Plots were created in Stata 13.0.

\section{RESULTS}

Patients and follow-up. Between March 2002 and December 2007, 241 patients were enrolled. Three patients were excluded from the intention-to-treat population. One patient was excluded due to evident brain metastases, one patient had no data ever recorded, and a third patient was randomised twice under two different ID numbers. Two-hundred and thirty-eight patients were included in the intention-to-treat analysis of eligible patients, 82 in the tamoxifen arm and 156 in the chemotherapy arm (Figure 1). Of the patients randomised to chemotherapy, 70 were allocated to weekly paclitaxel and 86 to PLD. Five patients did not receive the allocated treatment: two patients in the chemotherapy arm and three allocated to tamoxifen. Thus, 233 patients were included in the safety analysis. Baseline characteristics are summarised in Table 1. The CONSORT diagram for the population analysed for quality of life is shown in Supplementary Figure S1. The compliance rates with HRQoL assessments in the tamoxifen arm were $85 \%$ (47 of 55 ) and $72 \%$ (23 of 32 ) at weeks 8 and 16 , respectively. In the chemotherapy arm, these rates were $89 \%$ (119 of 133 ) and $77 \%$ (65 of 84 ), respectively. For analysis of QAPFS, 2 patients had no QoL information $12+2$ weeks prior to PD and thus had QoLs included 4 and 10 days after PD. Median follow-up was 169 weeks (range: 0-326).

Toxicity. There was a statistically significant difference in nonhaematological and haematological toxicities between the treatment arms with more nausea, mucositis, motor neuropathy, neurosensory, PPE, anaemia, leukopenia and neutropenia (Supplementary Table S1) in the chemotherapy arm. Two patients had febrile neutropenia in the chemotherapy arm, none in the tamoxifen arm. Patients on PLD had significantly more nausea, mucositis and PPE, but less anaemia compared with paclitaxel. In the chemotherapy arm, $4(2.6 \%)$ patients received G-CSF and 12 patients $(7.8 \%)$ experienced a dose reduction. Of the four dose reductions on paclitaxel, three were due to haematological toxicity (neutropenia and thrombocytopenia). On PLD, eight patients had a reduction in dose, seven due to non-haematological toxicity (PPE) and one due to haematological reasons (neutropenia). In 37 patients (24\%) chemotherapy was delayed for at least one cycle. In the tamoxifen arm, eight patients $(10.1 \%)$ had a loss in treatment days, ranging from 1 to 52 days.

Deaths on study. Ten (6.4\%) patients died in the chemotherapy arm; seven of those were related to the disease. One patient died due to sepsis of unknown origin, and one developed headache and became unconsciousness before she died. No autopsy was performed. The third patient was admitted to hospital with a subarachnoid haemorrhage and died the next day. Eight (10.1\%) deaths occurred on tamoxifen, with seven being disease related. One patient died of sepsis of unknown origin.

Efficacy. A total of 203 patients (85.3\%) had progressive disease during follow-up. Median PFS in the tamoxifen group was 8.3 weeks (95\% CI, 8.0-10.4) compared with 12.7 weeks (95\% CI,
Table 1. Baseline characteristics

\begin{tabular}{|c|c|c|c|c|}
\hline \multirow[b]{2}{*}{ Characteristics } & \multicolumn{2}{|c|}{$\begin{array}{l}\text { Chemotherapy } \\
\qquad(N=156)\end{array}$} & \multicolumn{2}{|c|}{$\begin{array}{l}\text { Tamoxifen } \\
(N=82)\end{array}$} \\
\hline & $\begin{array}{l}\text { No. of } \\
\text { patients }\end{array}$ & $\%$ & $\begin{array}{c}\text { No. of } \\
\text { patients }\end{array}$ & $\%$ \\
\hline \multicolumn{5}{|l|}{ Age, years } \\
\hline $\begin{array}{l}\text { Mean } \\
95 \% \mathrm{Cl}\end{array}$ & $\begin{array}{c}61.7 \\
(60.3,63.1)\end{array}$ & & $\begin{array}{c}62.1 \\
(60.1,64.1)\end{array}$ & \\
\hline \multicolumn{5}{|l|}{ FIGO stage } \\
\hline I & 11 & 7 & 5 & 6 \\
\hline ॥ & 7 & 4 & 1 & 1 \\
\hline III & 98 & 63 & 55 & 67 \\
\hline IV & 40 & 26 & 21 & 26 \\
\hline \multicolumn{5}{|l|}{ Tumour type } \\
\hline Ovarian cancer & 136 & 87 & 76 & 93 \\
\hline Peritoneal cancer & 15 & 10 & 4 & 5 \\
\hline Tubal cancer & 5 & 3 & 2 & 2 \\
\hline \multicolumn{5}{|l|}{ Histology } \\
\hline Serous & 106 & 68 & 71 & 87 \\
\hline Mucinous & 6 & 4 & 0 & 0 \\
\hline Clear cell & 10 & 6 & 1 & 1 \\
\hline Endometrioid & 15 & 10 & 2 & 2 \\
\hline Undiff. & 5 & 3 & 2 & 2 \\
\hline Unclass. & 2 & 1 & 2 & 2 \\
\hline Other & 12 & 8 & 3 & 4 \\
\hline Unknown & 0 & 0 & 1 & 1 \\
\hline \multicolumn{5}{|l|}{ Grade } \\
\hline Well diff. & 3 & 2 & 9 & 11 \\
\hline Mod. diff. & 34 & 22 & 15 & 18 \\
\hline Poor diff. & 101 & 65 & 49 & 60 \\
\hline Unknown & 17 & 11 & 9 & 11 \\
\hline Missing & 1 & 1 & 0 & 0 \\
\hline \multicolumn{5}{|l|}{ Performance status } \\
\hline 0 & 96 & 62 & 43 & 52 \\
\hline 1 & 53 & 34 & 37 & 45 \\
\hline 2 & 7 & 4 & 2 & 2 \\
\hline \multicolumn{5}{|l|}{ Type of progression } \\
\hline Progression during treatment & 10 & 6 & 6 & 7 \\
\hline $\begin{array}{l}\text { Progression within } 3 \text { months of end } \\
\text { of treatment }\end{array}$ & 90 & 58 & 38 & 46 \\
\hline $\begin{array}{l}\text { Progression between } 3 \text { and } 6 \\
\text { months of end of treatment }\end{array}$ & 56 & 36 & 38 & 46 \\
\hline \multicolumn{5}{|c|}{ Number of previous lines of chemotherapy } \\
\hline 1 & 86 & 55 & 52 & 63 \\
\hline 2 & 53 & 34 & 18 & 22 \\
\hline$>3$ & 17 & 11 & 12 & 15 \\
\hline
\end{tabular}

9.0-16.3) in the chemotherapy arm, corresponding to a HR of 1.54 (95\% CI, 1.16-2.05; log-rank $P=0.003$ ) (Figure 2). There was no statistically significant difference in PFS between the two chemotherapy arms (HR of 1.21, 95\% CI: 0.85-1.72).

By the end of follow-up, 204 (85.7\%) patients had died. Median OS time was 44.9 weeks (95\% CI, 31.1-57.7) in the tamoxifen arm and 46.9 weeks (95\% CI, 37.1-51.0) in the chemotherapy arm, corresponding to a HR of 1.10 (95\% CI, 0.82-1.47; log-rank $P=0.53$ ) (Figure 2). Stratification for type of prior progression did not affect the estimates of PFS or OS.

Two hundred patients (84\%) (136 in the chemotherapy arm and 64 in the tamoxifen arm) were evaluable and assessed for response. Of those, 2 patients (1.5\%) experienced complete response and 21 (15.4\%) partial response in the chemotherapy arm. In the tamoxifen arm, the best response obtained was partial response in three patients (4.7\%). Stable disease was achieved in 52 patients $(38.2 \%)$ in the chemotherapy arm and $20(31.3 \%)$ in the tamoxifen arm. Progression was reported in $61(44.9 \%)$ and 41 patients $(64.1 \%)$ in the chemotherapy arm and tamoxifen arm, respectively. 

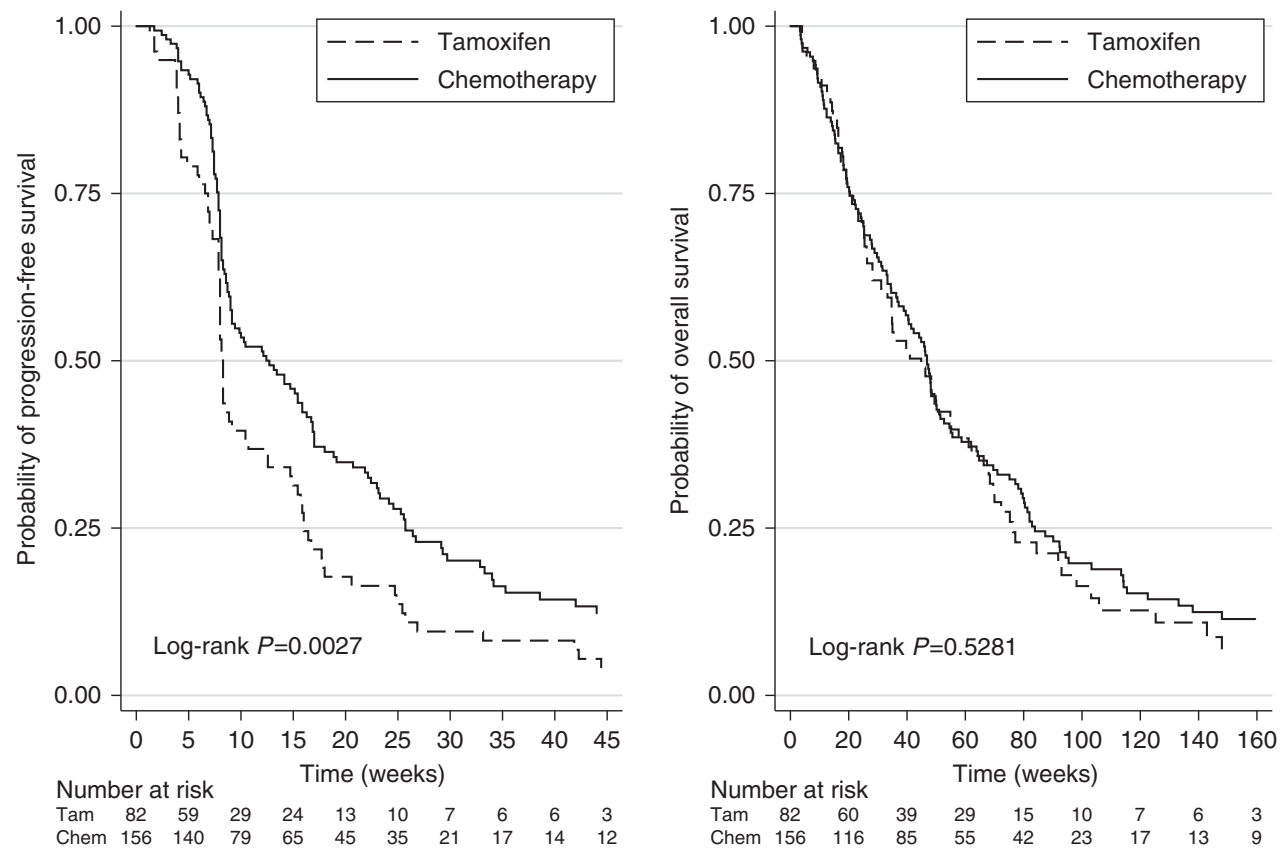

Figure 2. Kaplan-Meier estimates of progression-free and OS according to treatment arm.

Mean quality-of-life scores over time adjusted for the baseline. Over the study period, there was no statistical difference between the two arms for all the functioning scales (Figure 3). A statistically significant difference between the two arms existed at week 16 only for the abdominal GI symptom $(P=0.04)$ and hormonal side effects $(P=0.01)$ subscales, with more symptoms in the tamoxifen arm. Chemotherapy side effects symptoms were more common in the chemotherapy arm; however, the difference did not reach statistical significance.

Patient response analysis. Figure 4 illustrates the responder analysis (improved, stable or worsened) for each HRQoL scale and at week 8 based on a change of 15\%. In the chemotherapy arm, a significantly larger proportion of patients experienced a worsening in their social functioning at week 8 . For a change in HRQoL of $10 \%$ significantly more patients reported a worsening in both their social and physical functioning on chemotherapy compared with patients on tamoxifen (Supplementary Figure S2). The majority, 200 (90.9\%), reported abdominal symptoms at baseline with a median symptom score of 22.2. There was no difference in the proportion of patients who reported improvement of their GI symptoms between the treatment arms. There was further no statistical significant difference between the treatment arms in the proportion of patient with symptom improvement in subgroups of more or less GI symptoms at baseline.

Quality-of-life adjusted survival. Table 2 includes the qualityadjusted survival estimates for PFS. There was a significant difference between the utilities in favour of tamoxifen. However, this difference was offset by the difference in PFS and thus there was no difference in quality-adjusted PFS between the treatment arms $(P=0.14)$.

\section{DISCUSSION}

In this study of patients with PROC, single-agent chemotherapy was more effective in prolonging PFS compared with tamoxifen, but this small gain was traded off against poorer quality of life and increased treatment-related toxicity. Treatment with chemotherapy did not yield better control over GI symptoms compared with tamoxifen. There was no difference in OS between the treatment arms.

Our survival data in patients treated with single-agent chemotherapy are disappointing but in line with most previous reports (ten Bokkel Huinink et al, 1997; Gordon et al, 2001; Mutch et al, 2007; Vergote et al, 2010). Only about $17 \%$ of the evaluable patients showed complete or partial response on chemotherapy. Similar response rates have previously been reported for tamoxifen in PROC (Markman et al, 1996). Tamoxifen has not previously been directly compared with single-agent chemotherapy in PROC. A randomised study on the alkylating agent treosulfan and the $\mathrm{GnRH}$ (gonadotropin-releasing hormone) analoga leuprorelin reported no objective response in either of the treatment arms but significantly longer PFS in the chemotherapy arm (17 weeks vs 10 weeks, $P=0.035$ ). In our study only $4.7 \%$ responded to tamoxifen. Even though chemotherapy yielded better response rates than tamoxifen, the median PFS just exceeded 3 months. Patients on chemotherapy experienced more haematological and non-haematological toxicities and a deterioration of their social and physical functioning during treatment. We also studied the potential palliative effect of chemotherapy on GI symptoms as HRQoL data from second-line treatment with platinum-based chemotherapy suggested better symptomatic control especially in symptomatic patients (Brundage et al, 2012). The proportion of patients reporting GI-symptom improvement here was the same in both treatment arms, also in patients with higher symptom burden at baseline, suggesting that single-agent chemotherapy is not better than tamoxifen in palliating these symptoms. There is evidence that patients can accept a more toxic regimen if a benefit in survival is expected (Lee et al, 2013). In this study, we tried to assess these trade-offs by analysing QAPFS. There was no difference in QAPFS between the treatment arms, which may mean that the small gain in PFS was traded off against the poorer quality of life on chemotherapy.

The strengths of this study include its randomised design, the assessment of response and progression by RECIST criteria and the high completeness rate of HRQoL data until progression. There are several limitations of this study. The analysis of the primary end point of this study, HRQoL, was poorly defined in the protocol. Still, the primary and prespecified research question was to 

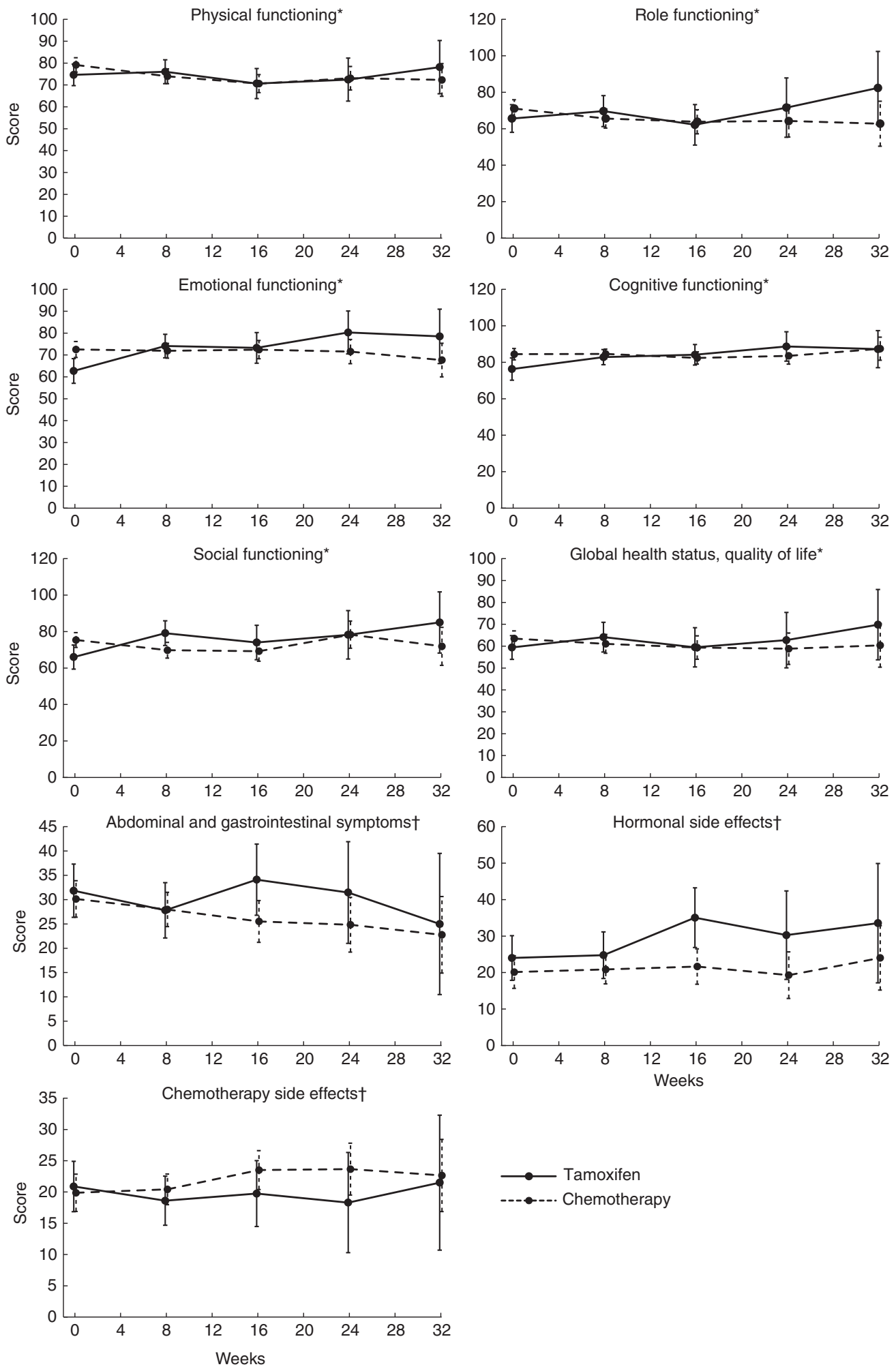

Figure 3. Baseline and scores from a mixed model for health-related quality of life function scales in both treatment arms. Error bars represent 95\% confidence interval for the estimated mean values. *Subscales derived from the EORTC_QLQ-C30; 'Subscales derived from EORTC-OV-28.

evaluate quality of survival with either of the regimens. We can therefore interpret the effects of the regimens on the quality-of-life outcomes as assessed here but need to be cautious to conclude from analyses of QAPFS as they may be underpowered. The higher completion rate of HRQoL assessments in the chemotherapy arm may also have biased our results. There was no evaluation of hormone receptor status and better patient selection to endocrine treatment may have led to better outcomes on tamoxifen treatment (Hatch et al, 1991). Also, the time to first response assessment may have been too short to observe an antiprofilerative effect. In the chemotherapy arm patients with unfavourable baseline characteristics were overrepresented. The patients had more previous lines of chemotherapy and more mucinous and clear-cell tumour histologies, which are known to be less chemosensitive compared 

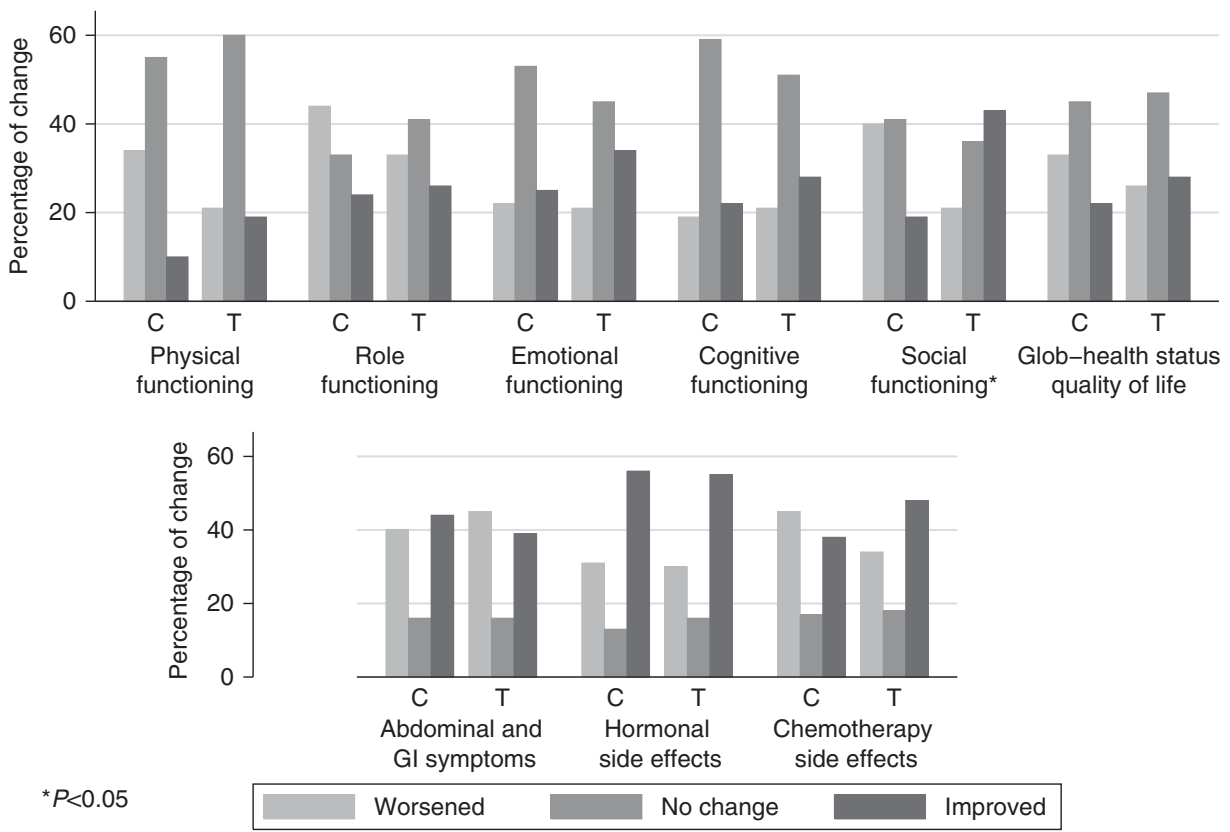

Figure 4. HQOL response proportions at 8 weeks (15\% cutoff).

Table 2. Quality-adjusted survival

\begin{tabular}{|c|c|c|c|}
\hline & \multicolumn{3}{|c|}{ QA-PFS ( $n=221(93 \%$ total population)) } \\
\hline Tamoxifen & 13.04 & 0.61 & $8.01(0.939)$ \\
\hline Diff (Tamoxifen - Chemotherapy) & -4.30 & 0.05 & -1.75 \\
\hline $95 \% \mathrm{Cl}$ & $(-8.54,-1.41)$ & $(0.01,0.09)$ & $(-4.350 .25)$ \\
\hline
\end{tabular}

with other histologies. These differences may have contributed to the slightly poorer results when compared with the results in the chemotherapy arm of the Aurelia study (Pujade-Lauraine et al, 2014). We could not demonstrate any benefit in OS for patients treated with chemotherapy probably in part due to the allowance of crossover between the trial arms post-progression. The tamoxifen and chemotherapy arms, 77 and $62 \%$, respectively, received further chemotherapy. Subsequent treatment after progression may also have had an impact on our estimates of HRQoL at progression as these data in a minority of patients were collected after progression.

Since the study was designed, treatment strategies of platinumresistant disease have evolved. The combination of single-agent chemotherapy with bevacizumab has been shown to increase response rates and PFS compared with single-agent chemotherapy alone, also with a benefit for GI symptoms (Pujade-Lauraine et al, 2014; Stockler et al, 2014). There was also a marginally significant benefit in OS in patients who had received paclitaxel plus bevacizumab (Poveda et al, 2015). However, due to the strict exclusion criteria with regard to the increased risk of GI perforation on bevacizumab, these findings may not be generalisable to all patients with PROC. Funding restrictions in many countries are another reason why single-agent chemotherapy is still widely used. There is also growing evidence that a subgroup of patients may again respond to platinum-based chemotherapy despite short disease-free survival. Especially patients with germline-BRCA mutation, but possibly also patients with somatic mutations in the DNA homologous repair pathway, may be more likely to respond to re-challenge with carboplatin and thus yield longer PFS (Alsop et al, 2012) compared with non-platinum based chemotherapy. Strategies targeting that pathway directly (i.e., PARP inhibitors) have also shown promising results in PROC (Kaufman et al, 2015) with a more favourable toxicity profile compared with chemotherapy.

In this phase III study in PROC a more toxic but presumably more effective treatment (single-agent chemotherapy) is compared with a potentially less toxic one (tamoxifen). The marginal longer PFS on chemotherapy was traded off against poorer quality of life questioning the palliative effect of single-agent chemotherapy. Our explorative analysis of QAPFS is suggested as a model to assess the trade-offs between prolonged PFS and QoL in this poor prognostic group of patients. The ongoing GCIG (Gynecologic Cancer Intergroup) symptom benefit study will hopefully provide more information on the benefit of chemotherapy on symptom control and quality of life (Friedlander et al, 2009). In this patient population with particularly poor prognosis, potential treatment strategies should also be assessed in terms of their benefit for QAS. Our analyses may serve as a model for further 
research on QAS estimates. Selection of patients based on molecular profiling may hopefully allow us in the future to better tailor management and ultimately improve survival in patients with PROC.

\section{ACKNOWLEDGEMENTS}

We acknowledge contribution of Dr Janne Kærn in patient recruitment and review of the manuscript. We also thank the staff of the data centre for data collection. Bristol Myers Squibb and Schering Plough provided an unrestricted educational grant for the study.

\section{CONFLICT OF INTEREST}

The authors declare no conflict of interest.

\section{REFERENCES}

Alsop K, Fereday S, Meldrum C, deFazio A, Emmanuel C, George J, Dobrovic A, Birrer MJ, Webb PM, Stewart C, Friedlander M, Fox S, Bowtell D, Mitchell G (2012) BRCA mutation frequency and patterns of treatment response in BRCA mutation-positive women with ovarian cancer: a report from the Australian Ovarian Cancer Study Group. J Clin Oncol 30(21): 2654-2663.

Batra S, Iosif CS (1996) Elevated concentrations of antiestrogen binding sites in membrane fractions of human ovarian tumors. Gynecol Oncol 60(2): 228-232.

Brundage M, Gropp M, Mefti F, Mann K, Lund B, Gebski V, Wolfram G, Reed N, Pignata S, Ferrero A, Brown C, Eisenhauer E, Pujade-Lauraine E (2012) Health-related quality of life in recurrent platinum-sensitive ovarian cancer-results from the CALYPSO trial. Ann Oncol 23(8): 2020-2027.

Buda A, Floriani I, Rossi R, Colombo N, Torri V, Conte PF, Fossati R, Ravaioli A, Mangioni C (2004) Randomised controlled trial comparing single agent paclitaxel vs epidoxorubicin plus paclitaxel in patients with advanced ovarian cancer in early progression after platinum-based chemotherapy: an Italian Collaborative Study from the Mario Negri Institute, Milan, G.O.N.O. (Gruppo Oncologico Nord Ovest) group and I.O.R. (Istituto Oncologico Romagnolo) group. Br J Cancer 90(11): 2112-2117.

Cancer Therapy Evaluation Program (1992) Common Terminology Criteria for Adverse Events (CTCAE) Version 1.0.

du Bois A, Meier W, Luck HJ, Emon G, Moebus V, Schroeder W, Costa S, Bauknecht T, Olbricht S, Jackisch C, Richter B, Wagner U (2002) Chemotherapy versus hormonal treatment in platinum- and paclitaxel-refractory ovarian cancer: a randomised trial of the German Arbeitsgemeinschaft Gynaekologische Onkologie (AGO) Study Group Ovarian Cancer. Ann Oncol 13(2): 251-257.

Fayers PM AN, Bjordal K, Groenvold M, Curran D, Bottomley A. on behalf of the EORTC Quality of Life Group (2001) The EORTC QLQ-C30 Scoring Manual. 3rd edn.BrusselsEuropean Organisation for Research and Treatment of Cancer.

Friedlander M, Butow P, Stockler M, Gainford C, Martyn J, Oza A, Donovan HS, Miller B, King M (2009) Symptom control in patients with recurrent ovarian cancer: measuring the benefit of palliative chemotherapy in women with platinum refractory/resistant ovarian cancer. Int J Gynecol Cancer 19(Suppl 2): S44-S48.

Gordon AN, Fleagle JT, Guthrie D, Parkin DE, Gore ME, Lacave AJ (2001) Recurrent epithelial ovarian carcinoma: a randomized phase III study of pegylated liposomal doxorubicin versus topotecan. J Clin Oncol 19(14): 3312-3322.

Gore ME, Fryatt I, Wiltshaw E, Dawson T (1990) Treatment of relapsed carcinoma of the ovary with cisplatin or carboplatin following initial treatment with these compounds. Gynecol Oncol 36(2): 207-211.

Hatch KD, Beecham JB, Blessing JA, Creasman WT (1991) Responsiveness of patients with advanced ovarian carcinoma to tamoxifen. A Gynecologic
Oncology Group study of second-line therapy in 105 patients. Cancer 68(2): 269-271.

Kaufman B, Shapira-Frommer R, Schmutzler RK, Audeh MW, Friedlander M, Balmana J, Mitchell G, Fried G, Stemmer SM, Hubert A, Rosengarten O, Steiner M, Loman N, Bowen K, Fielding A, Domchek SM (2015) Olaparib monotherapy in patients with advanced cancer and a germline BRCA1/2 mutation. J Clin Oncol 33(3): 244-250.

Lakusta CM, Atkinson MJ, Robinson JW, Nation J, Taenzer PA, Campo MG (2001) Quality of life in ovarian cancer patients receiving chemotherapy. Gynecol Oncol 81(3): 490-495.

Le T, Leis A, Pahwa P, Wright K, Ali K, Reeder B, Kinderchuck M, Ward K (2004) Quality of life evaluations of caregivers of ovarian cancer patients during chemotherapy treatment. J Obstet Gynaecol Can 26(7): 627-631.

Lee CK, Gebski VJ, Coates AS, Veillard AS, Harvey V, Tattersall MH, Byrne MJ, Brigham B, Forbes J, Simes RJ. Australia, New Zealand Breast Cancer Trials G (2013) Trade-offs in quality of life and survival with chemotherapy for advanced breast cancer: mature results of a randomized trial comparing single-agent mitoxantrone with combination cyclophosphamide, methotrexate, 5-fluorouracil and prednisone. Springerplus 2: 391.

Lortholary A, Largillier R, Weber B, Gladieff L, Alexandre J, Durando X, Slama B, Dauba J, Paraiso D, Pujade-Lauraine E. France Gg (2012) Weekly paclitaxel as a single agent or in combination with carboplatin or weekly topotecan in patients with resistant ovarian cancer: the CARTAXHY randomized phase II trial from Groupe d'Investigateurs Nationaux pour l'Etude des Cancers Ovariens (GINECO). Ann Oncol 23(2): 346-352.

Markman M, Iseminger KA, Hatch KD, Creasman WT, Barnes W, Dubeshter B (1996) Tamoxifen in platinum-refractory ovarian cancer: a Gynecologic Oncology Group Ancillary Report. Gynecol Oncol 62(1): 4-6.

Markman M, Rothman R, Hakes T, Reichman B, Hoskins W, Rubin S, Jones W, Almadrones L, Lewis Jr JL (1991) Second-line platinum therapy in patients with ovarian cancer previously treated with cisplatin. J Clin Oncol 9(3): 389-393.

McKenzie L, van der Pol M (2009) Mapping the EORTC QLQ C-30 onto the EQ-5D instrument: the potential to estimate QALYs without generic preference data. Value Health 12(1): 167-171.

Mutch DG, Orlando M, Goss T, Teneriello MG, Gordon AN, McMeekin SD, Wang Y, Scribner Jr. DR, Marciniack M, Naumann RW, Secord AA (2007) Randomized phase III trial of gemcitabine compared with pegylated liposomal doxorubicin in patients with platinum-resistant ovarian cancer. J Clin Oncol 25(19): 2811-2818.

Osoba D, Bezjak A, Brundage M, Zee B, Tu D, Pater J. Quality of Life Committee of the NC (2005) Analysis and interpretation of health-related quality-of-life data from clinical trials: basic approach of The National Cancer Institute of Canada Clinical Trials Group. Eur J Cancer 41(2): 280-287.

Poveda AM, Selle F, Hilpert F, Reuss A, Savarese A, Vergote I, Witteveen P, Bamias A, Scotto N, Mitchell L, Pujade-Lauraine E (2015) Bevacizumab combined with weekly paclitaxel, pegylated liposomal doxorubicin, or topotecan in platinum-resistant recurrent ovarian cancer: Analysis by Chemotherapy Cohort of the Randomized Phase III AURELIA Trial. J Clin Oncol 33(32): 3836-3838.

Pujade-Lauraine E, Hilpert F, Weber B, Reuss A, Poveda A, Kristensen G, Sorio R, Vergote I, Witteveen P, Bamias A, Pereira D, Wimberger P, Oaknin A, Mirza MR, Follana P, Bollag D, Ray-Coquard I (2014) Bevacizumab combined with chemotherapy for platinum-resistant recurrent ovarian cancer: The AURELIA open-label randomized phase III trial. J Clin Oncol 32(13): 1302-1308.

Sehouli J, Stengel D, Oskay-Oezcelik G, Zeimet AG, Sommer H, Klare P, Stauch M, Paulenz A, Camara O, Keil E, Lichtenegger W (2008) Nonplatinum topotecan combinations versus topotecan alone for recurrent ovarian cancer: results of a phase III study of the North-Eastern German Society of Gynecological Oncology Ovarian Cancer Study Group. J Clin Oncol 26(19): 3176-3182.

Stiggelbout AM, de Haes JC (2001) Patient preference for cancer therapy: an overview of measurement approaches. J Clin Oncol 19(1): 220-230.

Stockler MR, Hilpert F, Friedlander M, King MT, Wenzel L, Lee CK, Joly F, de Gregorio N, Arranz JA, Mirza MR, Sorio R, Freudensprung U, Sneller V, Hales G, Pujade-Lauraine E (2014) Patient-reported outcome results from the open-label phase III AURELIA trial evaluating bevacizumab-containing therapy for platinum-resistant ovarian cancer. J Clin Oncol 32(13): 1309-1316. 
ten Bokkel Huinink W, Gore M, Carmichael J, Gordon A, Malfetano J, Hudson I, Broom C, Scarabelli C, Davidson N, Spanczynski M, Bolis G, Malmstrom H, Coleman R, Fields SC, Heron JF (1997) Topotecan versus paclitaxel for the treatment of recurrent epithelial ovarian cancer. J Clin Oncol 15(6): 2183-2193.

Therasse P, Arbuck SG, Eisenhauer EA, Wanders J, Kaplan RS, Rubinstein L, Verweij J, Van Glabbeke M, van Oosterom AT, Christian MC, Gwyther SG (2000) New guidelines to evaluate the response to treatment in solid tumors. European Organization for Research and Treatment of Cancer, National Cancer Institute of the United States, National Cancer Institute of Canada. J Natl Cancer Inst 92(3): 205-216.

Vergote I, Finkler NJ, Hall JB, Melnyk O, Edwards RP, Jones M, Keck JG, Meng L, Brown GL, Rankin EM, Burke JJ, Boccia RV, Runowicz CD,
Rose PG (2010) Randomized phase III study of canfosfamide in combination with pegylated liposomal doxorubicin compared with pegylated liposomal doxorubicin alone in platinum-resistant ovarian cancer. Int J Gynecol Cancer 20(5): 772-780.

Williams C, Simera I, Bryant A (2010) Tamoxifen for relapse of ovarian cancer. Cochrane Database Syst Rev (3): CD001034.

This work is published under the standard license to publish agreement. After 12 months the work will become freely available and the license terms will switch to a Creative Commons AttributionNonCommercial-Share Alike 4.0 Unported License.

Supplementary Information accompanies this paper on British Journal of Cancer website (http://www.nature.com/bjc) 